\title{
41
}

\section{Integration of process planning and production management}

G. Halevi*, H.J.J. Kals**

* Technician -- Israel Institute of Technology, Dobnov St. 20 a

Tel-Aviv 64369 ISRAEL, Tel/Fax. + 97236962833

E-mail merhlev@techunix.technion.ac.il

**Director of the Center for Integrated Manufacturing \&

development, CIPV

University of Twente WB. Z.208 P.O. Box 2177500 AE

Enschede Tel. + 31534892534 Fax. + 31534893631

E-mail h.j.j.kals@utwpue.wb.utwente.nl

\begin{abstract}
New markets' demand objectives call for the integration of process planning in production management, which means that the process plan must be regarded as a variable, to be generated within seconds and with no human intervention. The matrix method, aims at introducing flexibility by integrating process planning and production management. The routing is presented by an Operation-Resources Matrix, which represents many possible routing, However, the selection of the routing is deferred to the moment of need. The method does a Material Requirements Planning (finite capacity) and Capacity Planning in one run, keeping the product network (Bill of Material) at all times. In this paper the concept of the matrix method is described.
\end{abstract}

Keywords

Process planning, MRP, MRPII, Production Planning, Capacity Planning, Scheduling.

\section{INTRODUCTION}

The Production Management (PM) task is to plan and produce the products according to management orders and policy. The major objectives of the task are:

- Meeting the delivery dates

- Keeping the capital, tied down in production, to a minimum

- Minimizing the manufacturing lead time 


\section{- Minimizing the idle times of the resources}

The traditional method to perform this task is divided into several stages (Hal, 1980). The orders are initially converted to required manufacturing activities, which defines what should be produced at the shop, and what should be purchased or outsourced. This stage is called MRP - Material Requirement Planning/Material Resource Planning. The material and the items that are made outside the company are taking care of by purchasing. The items that are produced in shop are handled in the next step: CP - Capacity Planning. In this step a long-range plan is made, planning the capacity required at each work center and helping to allocate the machines and manpower required to meet the goals of PM. The immediate short periods are scheduled in order to determine what jobs should be dispatched to the shop floor for execution in a certain time period. The Job Release (dispatch) step is the link between planning and execution. It initiates the production activities by the issue of orders to the shop floor, according to a short-term schedule taken from the capacity plan.

The main inputs to the PM are:

- The orders and tasks, including quantity and the delivery date

- The bill of materials for each product

- The routing for each component

- Inventory and resources

The planning and execution, in the traditional method, as described above, regards the routing as static and unalterable. Thus, the planning is simple, but it robs the shop from production flexibility and efficiency. [Seo and Egbelu 1996], [Hal, 1980]

Today's competitive market imposes new demands and objectives on the manufacturing process. Example of these demands is: delivery speed to market; product diversity and options; competitive product quality; customer satisfaction; and naturally, a competitive price. Since the traditional control methods do not supply solutions to these new demands, new methods and solutions are proposed. The main trend is to integrate technology with Production Management. Current Production Management often runs into problems such as: not meeting due dates, finite-capacity planning, overload, under-utilization and bottlenecks. Such problems occur mainly due to the fact that the routing (process plan) is regarded as unalterable. Anyone who has actually managed a production facility knows that this is not really the case. If the process routing is viewed as a variable, then many of the above problems can be solved by elimination. For example: An overload situation occurs in the Capacity Planning System. Traditional methods attempt to resolve this situation by transferring jobs to under-utilized periods or deferring operations to a later period. The result is increased Work In Process (WIP) and not meeting the due dates. By the dynamic routing method, this situation can often be solved by using a different routing that takes advantage of the under-utilized machines. Traditionally, internal due dates are set by the MRP system. They are not accurate as MRP considers infinite capacity. This causes unrealistic due dates, that in turn creates unrealistic capacity plans and schedules. 
With a dynamic system that can generate process plans within seconds without human intervention, there is no need to run the MRP system with infinite capacity. By employing a dynamic CAPP system the MRP system can be run considering finite capacity. These are only a few examples of how the use of a dynamic CAPP system may revolutionize production management procedures.

The manufacturing process is a dynamic one as resources change, machines fail, tools break, employees are unavailable, orders change, parts are rejected and reworked, power failures occur, etc. This dynamic nature calls for dynamic planning and flexibility of operation. Flexibility calls for immediate adaptation of process plans to the availability of resources. Furthermore, there are many optimization criteria for production planning and process planning. Each criterion calls for modification of the process plan. Thus a process plan should be dynamically adjusted to meet the criteria of the situation at hand.

This paper presents a dynamic system that integrates process planning with production management. The system integrates all stages, i.e. MRP, CP and SFC(shop flow control) with one dynamic logistics program. The dynamic and flexible behavior is obtained by employing the matrix method in process planning. The philosophy of this method is that a process planner is neither an economist nor a production planner. Therefore he should not make decisions, that is beyond his field of expertise. His task should be to prepare a matrix, covering all feasible routings, and subsequently let the expert in production planning decide which route to use, considering order size, plant load and shop floor state at the moment of decision making. The product tree (network) is being kept throughout all the planning steps.

\section{THE MATRIX PROCESS PLANNING CONCEPT}

The proposed CAPP system is composed of three stages: technology, transformation, and decision (mathematics). The technology stage generates Theoretical Processes (TP). The transformation stage constructs a matrix. The decision (mathematics) stage solves the matrix and generates a dynamic process plan according to the immediate requirements of the production facility.

The matrix represents an almost infinite number of possible routings. The decision of which routing to use is deferred to the time of execution. The routing decision making has been transformed from a technological into a mathematics decision. The decision-maker does not need to posses any technical knowledge, he has just to understand the capabilities of the matrix. The matrix solution time is less then a second.

The construction of the matrix has been discussed in several papers and a book (Hal 1995). In this paper, only the usage of the matrix is described.

The concept is based on the Operation - Machine matrix, where the entries are $\mathrm{Ti}, \mathrm{j}$ or $\mathrm{Ci}, \mathrm{j}$, depending on the requested criteria of optimization. Ti,j represents the time to perform operation $i$ on machine $\mathrm{j}$, and $\mathrm{Ci}, \mathrm{j}$ means the cost of performing operation $i$ on machine $j$. The matrix is shown in Table 1 . The sequence of entering operations into the matrix can be casual. The priority row indicates the constraints 
on the actual sequence of machining operations on a path. The number in this row indicates which operation must precede the considered operation. It does not have to follow this operation immediately. Priority number 0 means that this operation may be the first machining operation or be performed anywhere. For example, the priority of operation 010 is 000 ; the priority of operation 020 is 010 ; the priority of operation 030 is 020 ; the priority of operation 040 is also 010 . This means that the sequence of operations may be: $010 ; 020 ; 030 ; 040$ or $020 ; 030 ; 010 ; 040$, or 020 ; 010,$040 ; 030$.

Due to tolerance demands, some operations must be performed on the same machine or with the same fixture. These requirements are indicated in the row marked as relation.

Process planning is a technological decision-making process. By using the matrix method process planning is transformed from a technological into a mathematical process. The definition of the mathematical problem is as follows: Given a list of operations to be performed and a list of available facilities, a decision is required as to which machine (or machines) to use, which operation(s) to perform on each machine, what their sequence should be, and what cutting conditions to employ. The optimization criterion is either maximum production, or minimum cost. A penalty is added for extra time or expense, whenever a change in machine or set-up takes place. This penalty should cover the extra cost of: set-up, transfer of parts between machines, additional complications in capacity planning, job recording and inspection, etc. Note that the "penalty" is for a batch and thus is a function of the quantity to be produced. The larger the batch, the lower the penalty, and thus the higher the probability of selecting the best machine for each specific operation. The sequence (priority) and the relationship (relation) between operations must be maintained. That means that the sequence of operations may be altered, if by doing so the number of machine changes is reduced. However, the priority indicated in the matrix should be obeyed. This effect can be appreciated by reviewing Table 1 .

Table 1: The matrix format

\begin{tabular}{|c|c|c|c|c|c|c|c|}
\hline & $\begin{array}{l}\text { Oper\# } \\
010\end{array}$ & $\begin{array}{l}\text { Oper\# } \\
020\end{array}$ & $\begin{array}{c}\text { Oper } \\
\# 030\end{array}$ & $\begin{array}{c}\text { Oper } \\
\# 040\end{array}$ & $\begin{array}{l}\text { Oper } \\
\# 050\end{array}$ & $\begin{array}{c}\text { Oper } \\
\# 060\end{array}$ & Total \\
\hline Priority & 00 & 010 & 020 & 030 & 020 & 050 & \multirow[t]{3}{*}{ Cost Time } \\
\hline Relation & 0 & 0 & 0 & 0 & 2 & 0 & \\
\hline $\mathrm{TP}$ & 2.02 & 0.05 & 0.39 & 0.20 & 0.18 & 0.41 & \\
\hline \multicolumn{8}{|c|}{$\overline{\mathrm{C}}$} \\
\hline Mach \#1 & 12.48 & 4.60 & 5.96 & 5.20 & 5.12 & 6.04 & $\begin{array}{ll}39.40 & 9.85\end{array}$ \\
\hline Mach\#2 & 9.51 & 3.60 & 4.59 & 4.05 & 3.99 & 4.68 & $30.42 \quad 10.14$ \\
\hline Mach\#3 & 5.15 & 2.39 & 2.87 & 2.60 & 2.58 & -2.90 & $\begin{array}{lll}18.49 & 13.21\end{array}$ \\
\hline Mach\#4 & 999 & 999 & 999 & 1.86 & 999 & 999 & 999 \\
\hline Mach\#5 & 4.020 & 2.05 & 999 & 2.20 & -2.18 & 999 & 999 \\
\hline Macht16 & 6.54 & 2.00 & 3.28 & 2.90 & 2.86 & 3.32 & $21.50 \quad 10.75$ \\
\hline
\end{tabular}

Machine \#5;4.02+2.05+2.18=\$8.25=8.25 Min

Machine \#3;2.87+2.90=\$5.77=4.12 $\mathrm{Min}$ 


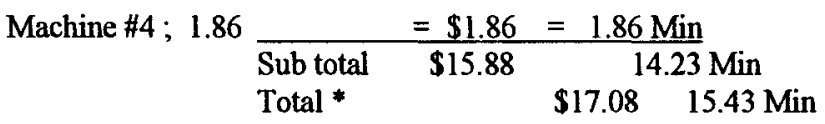

* Three penalties of $\$ 0.4$ each

By using an unaltered process and the best machine for each operation, as indicated by the shaded numbers, five penalties have to be added to the cost of the process. (Machine \# 5-6 - 3 - 4 - 5 - 3). However, the sequence of operation might be changed to the one shown by the arrows. (The penalty to use machine \#6 is greater than the machining cost difference between machine \#5 \& 6). In this case only two penalties have to be added to the cost of the process.

The solution for the maximum production (minimum time ) criterion (the upper matrix) results in only one machine (machine \#1) to perform all operations. The optimum process is a compromise between all the expenses and the individual operation cost. For solving this matrix problem, a special technique has been devised. The matrix solution is done within seconds.

Table 1 shows two optimum processes. The maximum production optimum will take 9.85 minutes and a cost of $\$ 39.4$. The minimum cost optimum will take 15.43 minutes and a cost of $\$ 17.08$. The difference between the two processes is, that for maximum production machine \#1 is selected, which is a powerful modern machining center that can perform all the operations in one chucking, while the minimum cost process uses rough, old, inaccurate machines for roughing operations and an accurate and stable machine for the finishing operations. Which process to select, depends on the shop floor load. For rush orders, the shortest production process is best, while in a slack situation the minimum cost alternative is the best. One can make such a decision only at the production management stage, and not at the process planning stage.

Currently the information transferred to production management contains usually the final decisions. For example : Use machine \#5 for $8.25 \mathrm{~min}$. (performing operations No. $010 ; 020 ; \& 050$ ). Next, use Machine \#3 for $4.12 \mathrm{~min}$. (performing operations No. 030; \& 050). Finally, use machine \#4 for $1.86 \mathrm{~min}$. (performing operation No. 040). Production management has to work with this information and subsequently will get into a lot of planning problems.

With the use of the matrix method, the process planner's task is to be a data provider and not a decision-maker. He only constructs the matrix. There is no optimum process that fulfills both the time and the cost optimum. One has to determine which one to use. Naturally, it is not a decision for the process planner to make.

The flexibility provided by the use of the matrix method, allows us to regard routing as a variable and not as a fixed one. Therefore, an improved production management method can be constructed. Such a method is proposed in the next section.

\section{THE MATRIX METHOD - GENERAL ARCHITECTURE}

The matrix method is based upon the following concepts: 
- Each decision is made by a qualified expert

- Each decision is based on real facts and not on assumptions

- Each decision is made at the time of execution, independent of the other decisions

- Each decision may be changed when circumstances change

- Keep the system simple

\subsection{Factory completed dates planning}

Traditional practice is that Production Management activities start with customer orders as input. Order delivery date is part of the order information. Delivery date plays a major role in PM stages. The MRP uses it for determining due dates for purchased and in house production orders. CP uses MRP due dates for in house machine loading. The complete date is one of the determining factors in establishing the quality of the shop performance. However, no one questions how the delivery date was determined. In many cases, sales promise non-realistic delivery dates, but PM regards them as a constraint.

In the proposed set up, the matrix system may be used by Sales and/or Management to establish realistic delivery dates. The matrix may be used as part of a larger control architecture as shown in Figure 1. The algorithm is as follows: the last machine capacity plan will be referred to as "load profile" (see Figure 7). The load profile is a result of a finite capacity machine loading process. The loading method will be described in section 3.3. The load profile shows the existing jobs planned on all the available resources. In any plan there might be some resource idle time. When a new order arrives, the matrix generates the best routing for the order. Considering the available inventory and the bill of materials, the system attempts to load the new order by using the idle spaces. This loading will be based on the minimum cost criterion (if the routing was generated for minimum cost optimization criteria). However as the order is loaded only at idle time or at the end of the loading period, the complete date might be far ahead. The matrix method may generate alternative processes, by using different machines. As an alternate process cannot be as efficient as the best process, the cost of producing the order will increase. But on the other hand, the idle time of the alternate machines might become shorter and the due date may become nearer. The matrix method has two features that might be handy in this case. One is the "Machine blocking option", were the scheduler is asked to ignore certain machines. The other is "Forced process planning option" were the machines to use are dictated externally. These features are used to generate alternatives. A list of alternative due dates and cost will be generated by the matrix method, as shown in Figure 2. Negotiation with the customer may be used to establish realistic delivery dates. 


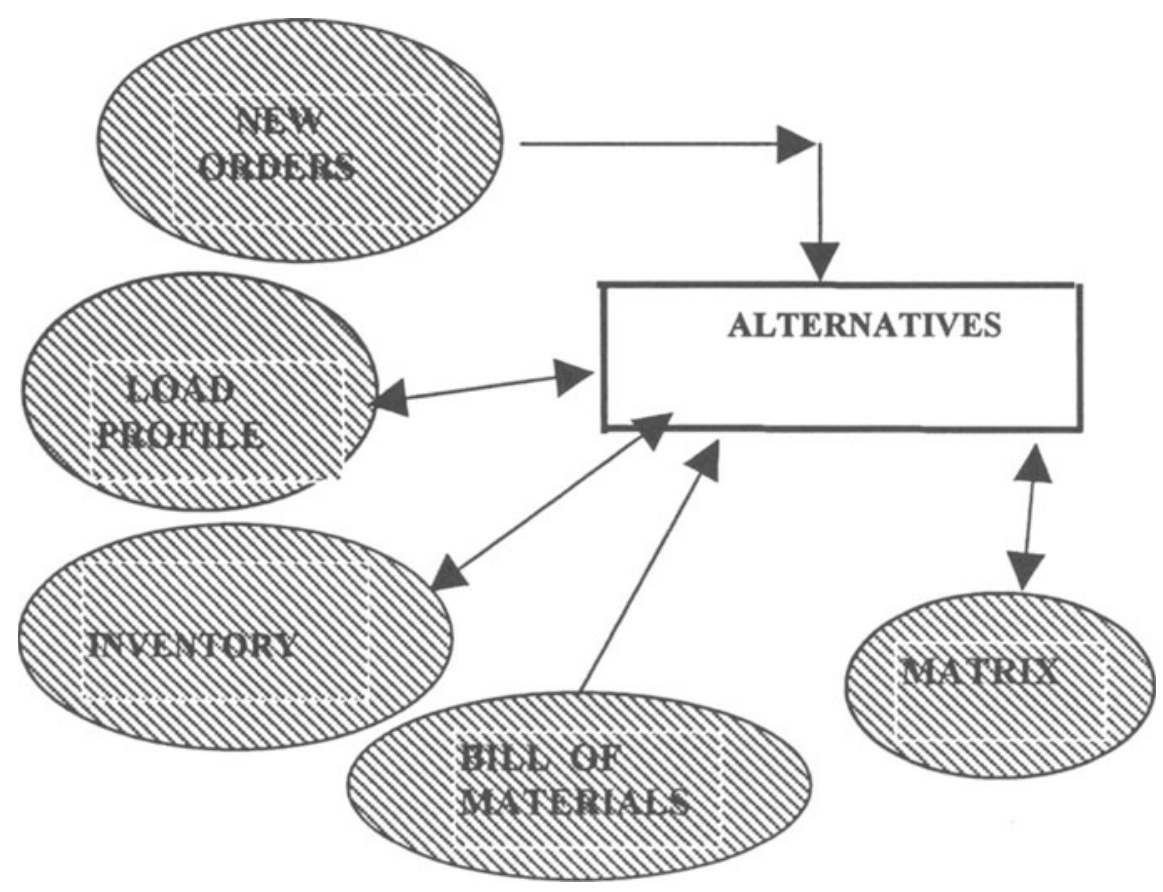

Figure 1 Control architecture using the matrix method

\begin{tabular}{|c|c|c|c|}
\hline \multicolumn{3}{|c|}{ ALTERN COST DUE DATE } & NOTES \\
\hline 1 & 30 & 120 & $\begin{array}{l}\text { New order super imposed on load profile } \\
\text { with minimum cost optimization }\end{array}$ \\
\hline 2 & 35 & 100 & $\begin{array}{l}\text { New order super imposed on load profile } \\
\text { with maximum production optimization }\end{array}$ \\
\hline 3 & 40 & 70 & With dynamic process plan \\
\hline $4-n$ & 46 & 68 & Several dynamic alternatives \\
\hline $\mathbf{n}-\mathbf{J}$ & 50 & 50 & Combinations of delaying existing orders \\
\hline
\end{tabular}

\subsection{Production Planning - Job Release}

The objective of this stage is to plan the activities on the shop floor, in order to meet delivery dates. The outcome of this planning is the order release to the shop floor for execution. In the matrix method, the order release must be practical. The planning is with finite capacity and with the use of the product tree. The detailed capacity plan has to be transformed into a real schedule.

The planning steps and method are as follows: 


\section{a. Determination of priorities}

For each order build a time-element tree, using data from the product structure. The tree is constructed from the order due-date backwards. Starting with the order, call the matrix indicating the item and the required quantity and retrieve the execution time. Transfer the total time to the time scale (in, let say, days) and subtract the time from the delivery date of the order. This yields the starting time for the job. Next, retrieve the following sub-assembly or component from the bill of materials and compute its required quantity. Call the matrix indicating the item name and the required quantity and follow the same procedure as above, calculate the starting time of that job. Continue the same procedure for all the items of the order and for all orders in the logbook.

b. Stock allocation.

The objective of this step is to allocate the available items in inventory to the available orders and adjust the early starting dates accordingly. It is done by scanning all the orders and finding the critical item i.e. the item where the starting day is the nearest one. Run over the tree of this item to find the product and the order. Search inventory and see if such product is available in stock. If available, allocate it to this order. Reduce the quantity of the order by the available quantity and rebuild the product tree with new quantities per item and new starting dates. In this case the starting time of the lowest level item will be changed and another order might become the critical one. Repeat the procedure of scanning all the orders to find the "new" critical item. In the case that there is no stock of the product, examine the stock availability for the next item using the same procedure.

c. Adjust quantities by economic considerations

Compute the EOQ - the Economic Order Quantity, either by the matrix method or by any other method or by external constraints. Split or compose orders if required, and recompile the product time base tree.

d. Capacity planning - machine loading

Machine loading is forward planning. The availability in time of each resource is symbolized by a row, were each slot in the row represents a planning period. The current date periods are shorter - say one hour- than the more distant periods, which each may cover two hours or more. The first ones, covering together a period of a few hours or a few days, depending on the kind of jobs and the average job time, serve as a scheduling period. The more distant slots, which together may take from a few hours up to weeks or months serve as a capacity planning period. When a job is allocated to the resource, the appropriate position(s) in the row hold(s) the order and the item code. Empty positions indicate idle resources. A critical planning path is defined as the path starting at the lowest independent item in the product structure with the earliest starting date, through its sub-assemblies up to the product. The critical path will have priority in machine loading. However, all the items for the sub-assembly have to be available before the assembly can start, i.e. all independent items that go into the critical sub-assembly will 
have a priority greater than the sub-assembly. The priority of such items is related to their starting date.

In the case of items for which the starting date falls in the past or which have a slack of $10 \%$ or more, the maximum production criteria is used for solving the matrix. (mind that the initial starting dates were established with the use of the cost criterion, which results in a longer processing time). For all others items, the minimum cost optimization criteria is used.

The loading procedure is as follows:

Scan all the orders and find the item with the nearest starting day. Run over the time-tree to find the product and the order. This order has the priority in machine loading. The loading is done from the lowest level item, from its last machining operation and forward. Subsequently, the matrix with the item name and quantity can retrieve a process. The retrieved process indicates the number of resources, the name of each resource and the time for the operation (including set-up and penalties). Start with the last operation, multiply its time by the quantity and divide by the period scale of the row. Determine how many periods are needed for this operation. Run over the row of the appropriate resource and search for an idle period. The search starts at the operation early start period (depends on the previous operations and the product tree). When the idle time is found, the name of the item is inserted in the row. In the case that the early available idle period is too far ahead of the early start period, the matrix will be called to generate an alternate process. The alternative process will attempt to reduce the waiting time by employing a different resource, which is idle at the required periods, and that it is economical to change. The initial proposed process is based on the minimum value of the matrix solution. However, in many cases the difference between the minimum value and the next higher value is negligible. Blocking the occupied resource (using the "machine blocking" feature) and resolving the matrix, will generate the next alternate routing. If a known machine is idle, the "forced process plan" feature will be used and the economics of using that process plan are examined. This process may be continued until the available space is found. Carrying out the planning actions as described above, results in a capacity plan, which includes a resource-utilizationoptimized schedule for the short-term periods.

e. Job release.

The capacity planning is a very important planning task, bridging the gap between long and short term planning. To carry out the production plan, jobs have to be released to the shop floor for execution. Different companies may have a different strategy regarding the length of the period for which the jobs are released to the shop floor. A long period might result in exceeding the delivery dates, while a short period might cause idle time at shop floor level. The auxiliary job dispatching strategy influences significantly the required length of the period. A period of 1-6 days is commonly used.

Before job execution can start, some auxiliary jobs have to be performed. Some of the auxiliary jobs might take a long period of time. One can 
distinguish two main strategies in dispatching auxiliary jobs. The first one, which is to be preferred when the execution of these jobs is relatively long when compared to the main jobs, deals with an early release during capacity planning. In this case, the main job is dispatched only after the auxiliary job has been reported ready. When the auxiliary job times are relatively short, one can dispatch these jobs at the beginning of the scheduling period.

\subsection{Execution}

The execution is divided into two stages:

1. Execution - auxiliary jobs

2. Execution - shop floor control

\subsubsection{Execution - auxiliary jobs}

The auxiliary jobs are: fixture design and construction, tool preparation, NCprogram generation, material preparation (inventory management and control), material handling (transport), quality control ( inspection), set-up instructions and set-up itself.

When jobs are assigned to or arrive at the medium range periods in the row, the auxiliary jobs attached to them are released. These jobs are given a due date situated before the scheduling or dispatching time of the main job. At this stage, the routing of the main jobs is known. However, the operators on the shop floor are allowed to alter the process when necessary to overcome disruptions. This means that the execution of the auxiliary jobs has to be flexible as well; otherwise the flexibility in routing cannot be realized. The matrix method can significantly contribute to the required auxiliary job flexibility.

The matrix contains all the required information about the process and its parameters. This information can be used to prepare an NC-program (G-functions). The TP in the matrix holds all the operation specifications. The resource-file contains the machine specifications, including the controller data. Constructing the matrix when the TP is known takes a few seconds. Therefore, with the matrix method no information is stored, it is created whenever needed. The same applies for the NC-program preparation from CL-Data. Such as NC-program can be generated at the instance of job allocation just before starting production. Nowadays, the possibilities to generate complete new NC-programs instantly are rapidly increasing.

Tools are assembled and preset in the tool room. Each tool is a accompanied by a certificate stating its dimensions. This information is used to offset the machine controller.

Set-up and job instructions are the result of the process selection made with the assistance of the matrix. Hence the matrix method can generate this data dynamically. Quality control is usually independent of the selected process. The same applies for material preparation. The TP process plan indicates alternative 
datum surfaces. These datum surfaces are used to design the fixture. At the release stage, the datum surfaces, the tool access constraints and the candidate machines are known. This information is transferred to the fixture design office. The information is considered during fixture design. The design might be based on the pallet concept, the modular concept or the Group Technology concept, in order to allow for the flexibility to transfer jobs from one machine to another.

\subsubsection{Execution - shop floor control}

Conditions on the shop floor are dynamic and occur when a machine fails, a tool breaks, employees are missing, orders change, parts are rejected and reworked, power failures take place, etc. So, a practical execution of the jobs, it must be allowed to deviate from the planned schedule. Therefore, the decision to release a job must take into account the immediate shop floor situation.

Figure 3 represents a schematic presentation of shop floor planning and control. The control architecture is based on the shop floor control architecture as presented by [Tiemersma, 1990].

The Scheduling function covers a short time slot of the capacity plan. The introduction of the scheduling module between capacity planning and dispatching enables the release of partial production orders. The production suborders can be merged or split before capacity planning on the cell level. The scheduling function extracts the required auxiliary jobs from the technical information, and checks their availability. Free operations are defined as the operations that are ready for immediate execution. The free operations list is transferred to dispatching for execution.

The Dispatching function is responsible for the release of jobs to the workstations. The dispatching concept includes, that whenever a resource is free, it searches for a job to be performed. If it is economical, a priority is given to the next operation of the same item. If not, the system scans the matrices of all parts in this particular machine column, and lists all free operations that the specific machine can do best. If the list is empty, a "look ahead" feature is used to determine when such an operation will become "free". The system will search for a free operation that the free machine can perform less efficiently, but economically. One method to compute the economics of using an "inefficient" operation, is to compute the difference in time between the "best" and the alternative operation time and comparing it to the time that the free machine will otherwise be idle. If such a free operation is found, it is allocated to the free machine. For more information see [Tiemersma, 1992] and [Hal, 1993]. 


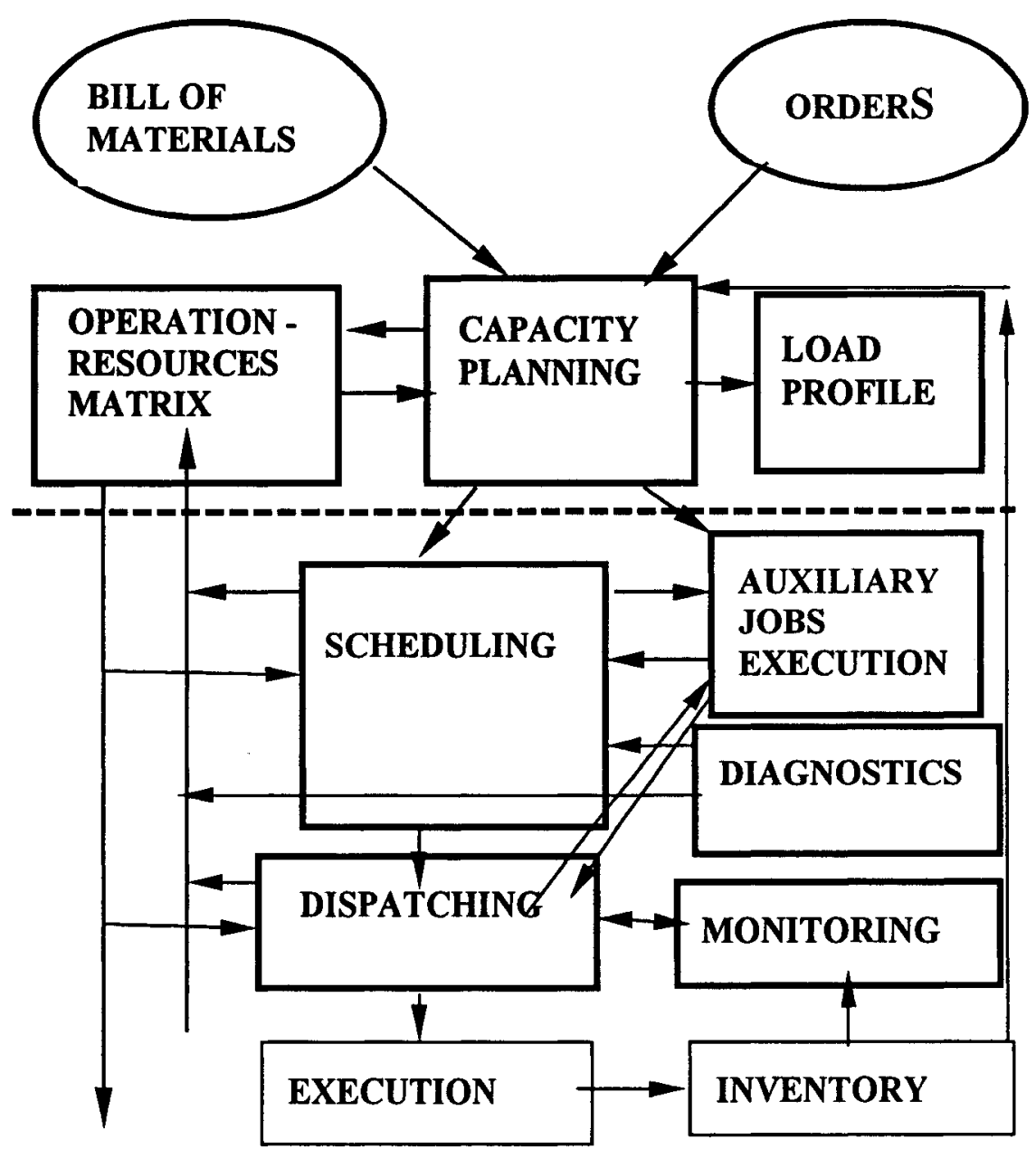

Figure 3 System architecture for Shop Floor Planning and Control

The Monitoring function is to collect the status of either a job, an operation or a workstation. For auxiliary jobs, the "released" phase is followed by the "completed" phase, to indicate that the related main job may be started. When a job is dispatched to a workstation, its status changes from "not released" to "released" and the first operation becomes a "free operation". When an operation is finished, the next operation becomes a "free operation". If the operation was the last operation on a job, the part is transferred to inventory and the job status is set to "complete". The status of the workstation normally changes between "idle" and "busy". The monitoring function informs the dispatching function of unexpected changes in status and of disruptions.

The Diagnostic function has to determine, in the case of disruptions, the consequences for the work plan. A delay of a job can have a snowball effect. In such a case of local disruption, the scheduling function will possibly have to 
generate an alternative work plan. In the case of a permanent disruption (due to improper routing), a permanent correction will be done on the matrix, in order to improve future work plans.

\section{THE MATRIX APPROACH - EXAMPLE}

To validate the feasibility of the proposed system and to check the execution time, a computer program has been prepared The program may handle several orders and parts. However, for simplicity and clarification of demonstration, two orders, 12 parts and 15 machines are considered in the example.

The assumed company makes two kind of cylinders: spring-return and double acting. Figure 4 shows the product.

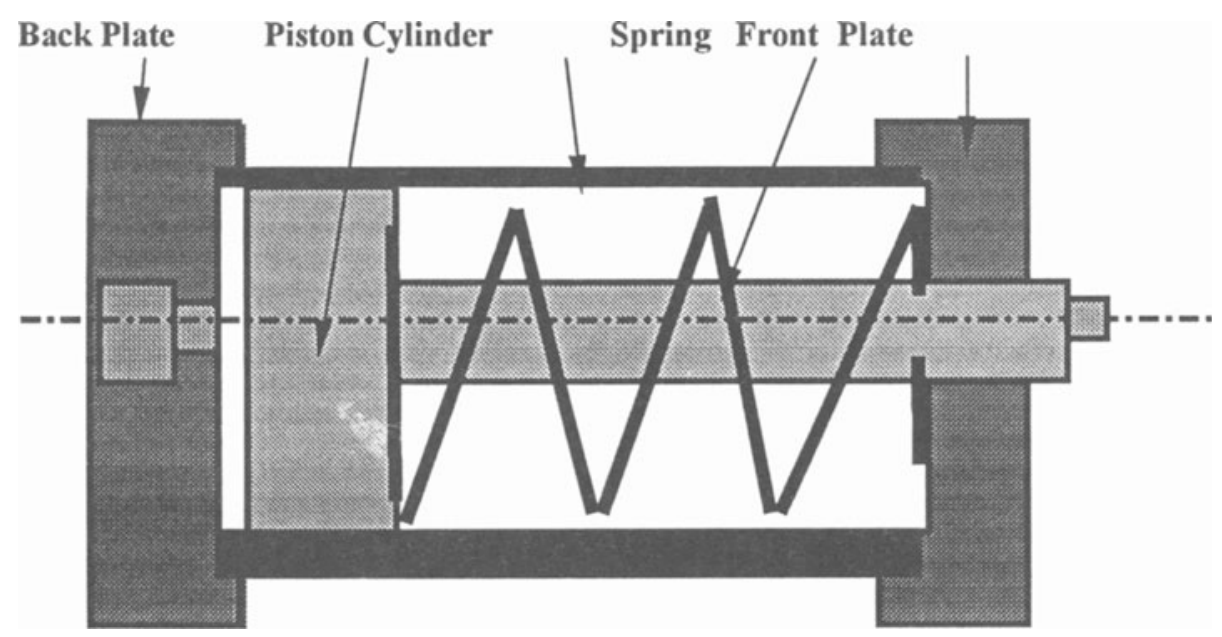

Figure 4 Pneumatic Cylinder Spring Return

The main menu of the computer program is used to choose: Order updating, Bill of material updating and Running mode. In the sample test two orders are introduced. They are: 80 spring return cylinders for delivery on day 40; and 40 double acting cylinder to be delivered on day 35 .

An TP process was made for each one of the cylinder items. An example of the TP for the front plate is shown in Table 2. The available 15 resources are detailed in Table 3. The TP process plan has been transformed into a matrix as shown in Table 4. The method used was detailed in section \#3. 
Table 2: TP Process for the Front Plate of the Spring Return Cylinder

\begin{tabular}{|c|c|c|c|c|c|c|c|c|c|}
\hline $\begin{array}{l}\mathrm{N} \\
\mathrm{o}\end{array}$ & Operation & $\mathrm{Pr}$ & $\begin{array}{l}\mathrm{T} \\
00 \\
1\end{array}$ & L & $a$ & Feed & $\mathrm{V}$ & $\overline{\mathrm{kW}}$ & $\begin{array}{l}\mathrm{T} \\
\min \end{array}$ \\
\hline 1 & Sawing & 00 & B & & & & & & 2.8 \\
\hline & & & & & & & & & \\
\hline 2 & Side milling & 10 & 50 & $\begin{array}{l}113 x \\
2\end{array}$ & 2.5 & 304 & 88 & 1.0 & 2.02 \\
\hline 3 & $\begin{array}{l}\text { Face milling - } \\
\mathrm{R}\end{array}$ & 10 & 80 & 76 & 1.59 & 1428 & 118 & 6.63 & 0.05 \\
\hline 4 & $\begin{array}{l}\text { Face milling - } \\
\text { F }\end{array}$ & 30 & 80 & 143 & $\overline{0.41}$ & 900 & 147 & 1.34 & 0.16 \\
\hline 5 & $\begin{array}{l}\text { Assembly } \\
\text { Holes }\end{array}$ & 20 & 10 & $20 \times 4$ & & 0.2 & 25 & 0.6 & 0.96 \\
\hline & & & & & & & & & \\
\hline 6 & Side milling & 10 & 50 & $\begin{array}{l}113 x \\
2\end{array}$ & 2.5 & 304 & 88 & 1.0 & 2.02 \\
\hline 7 & $\begin{array}{l}\text { Face milling - } \\
\mathrm{R}\end{array}$ & 10 & 80 & 76 & 1.79 & 1401 & 116 & 7.34 & 0.05 \\
\hline 8 & $\begin{array}{l}\text { Face milling - } \\
\mathrm{F}\end{array}$ & 70 & 80 & 143 & 0.21 & 370 & 164 & 0.37 & 0.39 \\
\hline 9 & Drill U $46 \varnothing$ & 10 & 44 & 4 & & 0.27 & 114 & 10.3 & 0.2 \\
\hline 10 & $\begin{array}{l}\text { Boring } 46 \varnothing- \\
\mathrm{Mr}\end{array}$ & 90 & 40 & 4 & & 804 & 93 & 0.22 & 0.18 \\
\hline 11 & $\begin{array}{l}\text { Boring } 46 \varnothing- \\
\text { Mf }\end{array}$ & 10 & 40 & 4 & & 359 & 106 & .07 & 0.41 \\
\hline 12 & piston hole & 11 & 16 & 16 & & & & 2.0 & 0.34 \\
\hline & & & & & & & & & \\
\hline
\end{tabular}

The steps of the module are as follows:

a. Product Bill-of-Material Explosion - Quantity and Time.

The objective of this step is to set priorities for stock allocation. It works from the delivery date of each order backward. It starts with the assembly of order number one, i.e. item \#1. The matrix is called to generate a process with minimum cost criteria, for the assembly of 80 items of product \#1. The output of the matrix module gives the total time, and the detailed operations, including the time on each individual machine. The total time is multiplied by the quantity, and translated into days. The duration in days of the assembly product \#1 is subtracted from the delivery date. The result is the starting date of the assembly (day 39.10). The next item on the Bill-of-Materials (see Figure 5) is the Back Plate item \#2. The quantity per unit assembly is 1. Therefore the quantity of item \#2 is 80 . The matrix is called to generate a process with minimum cost criteria, for processing 80 pieces of item $\# 2$. The 
output of the matrix module gives the total time and the detailed operations, including the time on each individual machine. The detailed operation data are used to generate a report on the load of each machine, indicating starting and ending days of each item on each machine and the total load on each machine. This report is for general use only. The total time is multiplied by the quantity, and translated into days. The duration in days required to produce item $\# 2$ is subtracted from the starting date of the assembly, item \#1, and sets the starting day for item \#2 (day 36.43). This process continues till the last item of the second order has been dealt with. (in total 20 retrievals from the matrices, which took 3 seconds on a $33 \mathrm{MHz}$ computer). A summary report is given in Figure 5. The bold numbers indicate the starting date of each item. The number in brackets indicates the quantity of each item whose number is given as (\#).

Table 3: The Available Machines

\begin{tabular}{|l|l|l|l|l|l|}
\hline $\begin{array}{l}\text { Mac } \\
\text { No. }\end{array}$ & Machine description & $\begin{array}{l}\text { Power } \\
\text { kW }\end{array}$ & $\begin{array}{l}\text { Speed } \\
\text { RPM }\end{array}$ & $\begin{array}{l}\text { Handle } \\
\text { time }\end{array}$ & $\begin{array}{l}\text { Relat } \\
\text { cost }\end{array}$ \\
\hline 1 & Milling Machining center & 35 & 1500 & 0.10 & 4 \\
\hline 2 & Large CNC Milling & 35 & 1200 & 0.15 & 3 \\
\hline 3 & Manual milling machine & 15 & 1500 & 0.66 & 1.4 \\
\hline 4 & Small drill press & 1 & 1200 & 0.66 & 1 \\
\hline 5 & Old milling machine & 15 & 2400 & 1.0 & 1 \\
\hline 6 & Small CNC milling & 10 & 3000 & 0.25 & 2 \\
\hline 7 & CNC Lathe & 25 & 3000 & 0.15 & 3 \\
\hline 8 & Manual Lathe new & 15 & 3000 & 0.42 & 2 \\
\hline 9 & Manual Lathe old & 10 & 2400 & 0.66 & 1 \\
\hline 10 & Circular Saw & & & & 1 \\
\hline 11 & Band Saw & & & & 1 \\
\hline 12 & Hack Saw & & & & 1 \\
\hline 13 & Manual assembly & & & & 1 \\
\hline 14 & Machine assembly & & & & 1.5 \\
\hline 15 & Robotics assembly & & & & 3 \\
\hline
\end{tabular}

b. Stock allocation.

The objective of this step is to allocate available stock and to adjust the quantities of each item on the ordered products. The allocation is carried out with the product tree and gives priority to the critical path. The program scans all paths of the Bill-of-Material trees in a search for a low-level item of which the starting day is the nearest one. In this example, as indicated in Figure 5, item \#7 of order 2 has a starting date of 31.18 , which is the nearest one. 
Table 4 : The Time Matrix Content

\begin{tabular}{|c|c|c|c|c|c|c|c|c|c|c|c|c|c|c|c|c|}
\hline $\mathrm{OP}_{\mathrm{p}}$ & Pri & $\begin{array}{c}M \# \\
1\end{array}$ & $\begin{array}{c}\mathrm{N} / \mathrm{k} \\
2\end{array}$ & $\begin{array}{c}M= \\
3\end{array}$ & $\begin{array}{c}114 \\
4\end{array}$ & $\begin{array}{c}11 \% \\
5\end{array}$ & $\begin{array}{c}.1 \%= \\
6\end{array}$ & {$\left[\begin{array}{c}11 \neq \\
7\end{array}\right.$} & $\begin{array}{c}1 \% \\
3\end{array}$ & $\begin{array}{c}1+1 \\
9\end{array}$ & $\mid \begin{array}{l}11 ; \\
10\end{array}$ & $\left|\begin{array}{c}14 \\
11\end{array}\right|$ & $\mid \begin{array}{l}\mathrm{N}=4 \\
12\end{array}$ & \begin{tabular}{|c|}
$M 1 *$ \\
13 \\
\end{tabular} & \begin{tabular}{|c|}
$\mathrm{M}$ \\
14
\end{tabular} & \begin{tabular}{|l|l}
115 \\
15 \\
\end{tabular} \\
\hline$B$ & $A$ & C & K & & & $\vec{p}$ & $\mathrm{~L}$ & $A$ & $\bar{T}$ & $\bar{E}$ & & 44 & & & & \\
\hline 10 & (ini) & 99 & $y+1$ & 99 & w? & (x) & 14) & $(\mu)$ & (4) 1 & 10,1 & $17 x$ & $\operatorname{lin} 1$ & $12: 1$ & 99 & 991 & $1 x$ \\
\hline B & $A$ & C & $K$ & & & $\mathrm{p}$ & $\mathrm{L}$ & A & $T$ & $E$ & & $\$ 3$ & & & & \\
\hline 10 & $\infty 0$ & 312 & 3.17 & $36 \times$ & 94 & t112 & 3.27 & (y) & 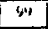 & ; & 94 & ") & $9 \%$ & $\%$ & (n) & in \\
\hline 20 & 10 & 0.15 & 12 & 1.71 & (W) & 2115 & 1.5 & 99 & $\overline{x i}$ & Wix & in & $\overline{91}$ & 可 & Wh & (W) & $m$ \\
\hline 30 & 20 & 1.26 & \begin{tabular}{|l|}
1.31 \\
\end{tabular} & 1.82 & (9) & (w) & 1.41 & $9 y$ & (w) & is) & (4) & 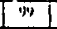 & 99) & wi & 4.9 & $w$ \\
\hline 40 & 10 & \begin{tabular}{|l|l}
1.37 \\
\end{tabular} & \begin{tabular}{|l|}
1.62 \\
\end{tabular} & 2.13 & 3.5 .1 & 245 & 17 & 99 & 9.) & 99 & is & p) & (v) & 9) & wi & 15 \\
\hline 50 & 10 & 2.106 & 211 & 2.62 & 1202 & $2.1 \%$ & 221 & 99 & 99 & in & i) & 2.) & 9) & $\%$ & 94) & is \\
\hline B & A & C & $K$ & & & P & L & A & $T$ & $E$ & & $\# 2$ & & & & \\
\hline 10 & 00 & 3.12 & 3.17 & 3.68 & $(x)$ & : & 327 & 91) & 9) & 94 & W & 9 & 9 & $\eta$ & 74 & ir \\
\hline 20 & 001 & 0.13 & 1.2 & 1.71 & $\%$ & 2.03 & 13 & $y$ & 47) & yi & $\sqrt{4}$ & 01 & $\sqrt{5}$ & 9? & $y$ & in) \\
\hline 30 & 20 & 1.54 & 1.53 & 2.115 & 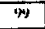 & 99 & 1.64 & \%) & Wo & 94 & कy & $\mathbb{N}$ & (w) & 97 & 9 & 7) \\
\hline 40 & 10 & 1.30 & 1.35 & 1.36 & 1.86 & 2.2 & 1.45 & 9) & 27 & 9i) & $4 \%$ & ?) & 99 & wis & 79 & "1" \\
\hline 50 & 40 & 123 & 133 & 1.94 & 99 & 218 & $1+3$ & 99 & 99 & iy & 91 & 94 & $9 y$ & 99 & (3) & w) \\
\hline 60 & 50 & 1.51 & $\begin{array}{ll}1.56 \\
\end{array}$ & 2.07 & $\%$ & $\%$ & 1.56 & wi & 99 & 99 & 91 & (*) & 99 & 91) & 99 & $\%$ \\
\hline$F$ & $\mathbf{R}$ & 0 & $N$ & $T$ & & $P$ & L & A & $\mathrm{I}$ & $E$ & & $\# 7$ & & & & \\
\hline 101 & 0 & 9 & 49 & $\begin{array}{ll}\infty \\
\end{array}$ & (x) & 99 & (4) & $m$ & 99 & 9y & 7.81 & 661 & 12.2 & pr) & w) & \% \\
\hline$E$ & $\mathbf{R}$ & 0 & $\pi$ & $T$ & & $P$ & L & $A$ & $T$ & $\bar{E}$ & & $\# 6$ & & & & \\
\hline 10 & 0 & 3.12 & 3.17 & $3.6 \times 1$ & 97 & 502 & 3.78 & 99 & $\overline{9 y}$ & (9) & 49 & 9 & 9 & 99 & (4) & 9) \\
\hline 20 & 10 & 1.15 & 1.2 & 8.711 & 24 & 3015 & 1.3 & 9 & $9 \%$ & $\sqrt{39}$ & gin & 49 & 99 & 99 & 34 & $\sqrt{94}$ \\
\hline 30 & 20 & 1.26 & 1.31 & $1.32 !$ & $\%$ & w & $1 .+1$ & 19 & xy & \%) & (क) & का & $\overline{94}$ & .9 & (in) & in \\
\hline 40 & 101 & $3 \sin$ & 2.11 & 2.62 & $2 \div 2$ & $2 \%$ & $2=21$ & $m$ & r) & $m 1$ & (4.) & 9) & 94 & 94 & $\sqrt{4}$ & 6 \\
\hline$F$ & $\mathbf{R}$ & 0 & $N$ & $T$ & & $P$ & $\mathrm{~L}$ & $d$ & $I$ & $E$ & & $\ddot{\pi 5}$ & & & & \\
\hline 10 & 0 & 3.12 & 3.17 & 3.681 & ay & $+1,12$ & $3: 7$ & 99 & 99) & 94 & Yy & 99 & 9 & yy & (w) & (y) \\
\hline 20 & 0 & 0.15 & 1.2 & 1.71 & w) & 2.115 & 1.3 & (9.) & $\%$ & 54 & $9 y$ & 49 & 99 & 49 & (9) & W \\
\hline 30 & 20 & 1.54 & 0.53 & 2.155 & 79 & W & 164 & g) & 99 & 94 & 9 & W' & (4) & 99 & N. & 49 \\
\hline 40 & 10 & 1.31 & 0.35 & 1.00 & $1.3 \mathrm{~s}$ & 22 & 1.55 & (9) & 9 & (n) & 49 & की & $9 n$ & (9) & 94 & (9) \\
\hline 50 & 50 & 1.28 & 1.31 & I..S? & $\%$ & 2.18 & $1 .+3$ & 9 & 9 & का) & $\sqrt{49}$ & 99 & 9 & 99 & (4) & $y$ \\
\hline 60 & 60 & 1.51 & 1.36 & 2.071 & 99 & $4 \%$ & 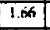 & $\%$ & 9 & 9r) & 99 & $\sqrt{*}$ & 99 & (y) & $\sqrt{9 y}$ & $\bar{w}$ \\
\hline 70 & 40 & 1.4 & (..4) & 1.01 & 9) & 94 & 1.501 & (4) & 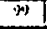 & פ') & (40) & $N$ & 99 & (4) & $\because 1$ & $\psi_{i}$ \\
\hline $\mathrm{P}$ & $I$ & $S$ & $T$ & 0 & $\mathrm{~N}$ & & & & & & & $\$ 9$ & & & & \\
\hline 10 & 0 & 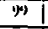 & 59 & क) & (N) । & $9 \%$ & sy & 2.03 & 2.33 & 250 & $y$ & n & (9) & $9 !$ & $m$ & (N) \\
\hline 20 & 10 & pr) & 9) & $\cdots 1$ & (w) & 叫 & 99 & 1.91 & 1.85 & $y=$ & & & & & & \\
\hline$P$ & $I$ & $S$ & $T$ & 0 & $N$ & & & & & & & $\mathrm{Ag}$ & & & & \\
\hline 10 & 01 & Wil & 49 & w.1. & , in? & $+\cdots$ & +41 & 1.15 & 1.21 & $i \% 1$ & (4) & का! & $(4)$ & $|x| ! \mid$ & ${ }^{n \prime !} !$ & 101 \\
\hline 201 & 101 & (19) & $\overline{401}$ & 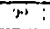 & $4 \%$ & 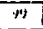 & $\begin{array}{ll}N^{2} \\
\end{array}$ & 1.15 & 1.21 & (int & 14 & $\sqrt{41}$ & wi 1 & $7 \%$ & $\sqrt{y i}$ & 194 \\
\hline$C$ & $y$ & i. & 1 & $\mathrm{~N}$ & $D$ & $E$ & $R$ & & & & & 71 & 0 & & & \\
\hline 101 & 01 & w' & $n 1$ & $n !$ & (n) ! & , & 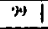 & $2 \times 1$ & $3: 1$ & 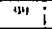 & $\cdots 1$ & 4) & $m 1$ & $m 1$ & $\cdots 1$ & $1+1$ \\
\hline$\Lambda$ & 5 & 5 & $E$ & .11 & B & 1 & $Y$ & & & & & +1 & $\&$ & $=11$ & & \\
\hline (10) 1 & 0 & 94 & 41 & *w & (4) & 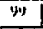 & $4+1$ & $w$ & (r) & 91 & (y) & $\%$ & ות) & 30 & $\$ 11$ & 8.0 \\
\hline
\end{tabular}

Following the Bill-of-Material tree, from this item onwards, indicates that this item is part of product \#11, order number 2 . Therefore, order \#2 is the critical one and stock allocation will start with this order. It starts with the high level items and proceeds towards the low-level items. Hence, initially product \#11 will search for available stock. In this example the available stock shows:

$\begin{array}{llllllll}\text { Item } & 1 & 2 & 3 & 5 & 8 & 10 & 11 \\ \text { Quantity } & 3 & 3 & 13 & 14 & 10 & 3 & 20\end{array}$

Therefore, the quantity for item \#11 will be reduced to 20 pieces. and the quantities of all items in the product tree will be adjusted accordingly. The matrix will be called to generate a process plan for the new quantity, in the same manner as before. The adjusted starting date of item \#7 is now 33.19, which is shown in Figure 6. Figure 6 shows an intermittent stage of the stock 
allocation module. The critical path is now item \#4 (32.90) of product \#1 in order \#1. Subsequently, product \#1 will search for available stock. There are 3 products of item \#1. Its quantity, and the quantity of all the items on the product tree will be adjusted. Next, the starting date will be recomputed. After the adjustment, the new lowest starting day is on item \#4 of product \#1 (33.15) and is on the critical path (33.15 is compared to 33.19 of item \#7 of product \#11). Following the product tree, brings us to item \#2 of product \#1 to become the critical one. The procedure continues till stock has been allocated to all items. This step took 5 seconds on a $33 \mathrm{MHz}$ personal computer.

c. Capacity Planning - Machine loading

An array mac $(15,400)$ was defined. The number ' 15 ' represents the resources as detailed in Table 3. The number ' 400 ' represents the periods. For this demonstration only 23 periods are used. The machine loading is forward planning. When a job is allocated to the resource, the appropriate positions in the row hold the order and item code. In this example the two digits are used: The first indicates the item number (as defined in Figure 5) and the second digit represents the order number. The critical path has priority in machine loading. To determine the critical order, the program scans all paths of the Bill-of-Material tree in a search for the minimum value. In this example, item \#4 of product \#1, order \#1 is the critical one. The matrix is called to generate a process for 74 units of item \#4 ( see Figure 7). As seen from Table 4, the process calls for 6.6 minutes on machine \#11. This time is multiplied by the quantity and divided by the period scale. As it is the first item to be loaded, it starts at period \#1. Figure 7 shows that machine \#11 are loaded at periods 1 \& 2 with 41 . ( item \#4 of order \#1). Next, the matrix is called to generate a process for 74 units of item \#3. The retrieved process calls for: machine \#5 for $6.07 \mathrm{~min}$., then for machine \#3 for $1.82 \mathrm{~min}$, ending with machine \#4 with 5.15 $\mathrm{min}$. (In consulting the reasoning of the generated process, remember that Table 4 gives the time, while the process is regarded for minimum cost). 


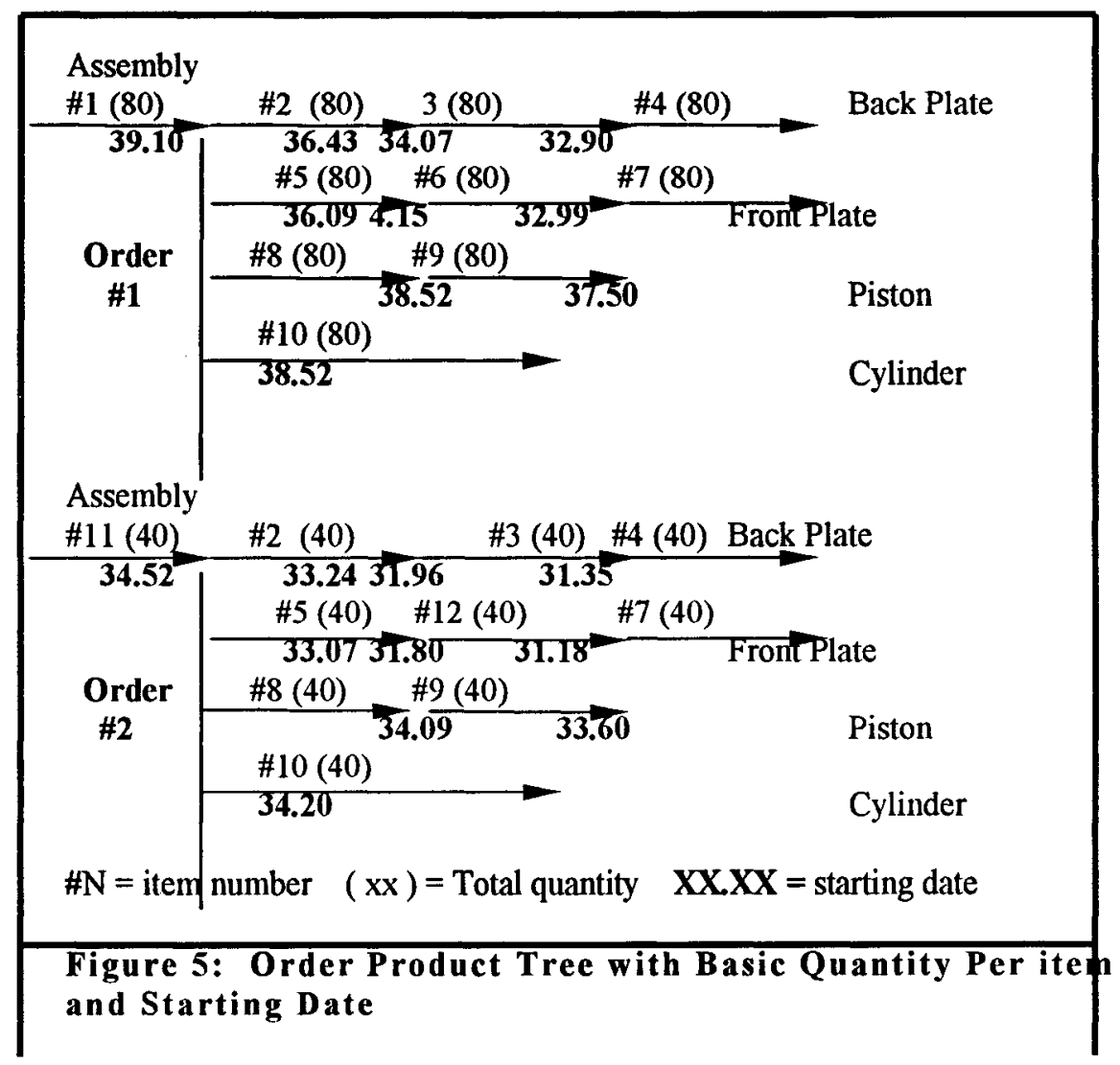

Machine loading starts with the last operation, in this case with machine \#4 for 2 periods. It may start only after item \#4 is finished, i.e. at period \#3. The array shows this through the symbol of " 31 " in the periods $3 \& 4$ on machine \#4. The next operation calls for 1 period on machine \#3 and then for 2 periods on machine \#5. This process continues for item \#2. As item \#1 is an assembly, it will start only after all the components are available. Therefore, machine loading will proceed to item \#7. Item \#7 can be assigned to period \#1, as it is an independent item. The matrix recommends a process of 6.6 minutes (2 periods) on machine \#11. However, the machine is occupied till period \#3; therefore it is loaded at periods \#4 \& \#5. Next, item \#6 is treated. 


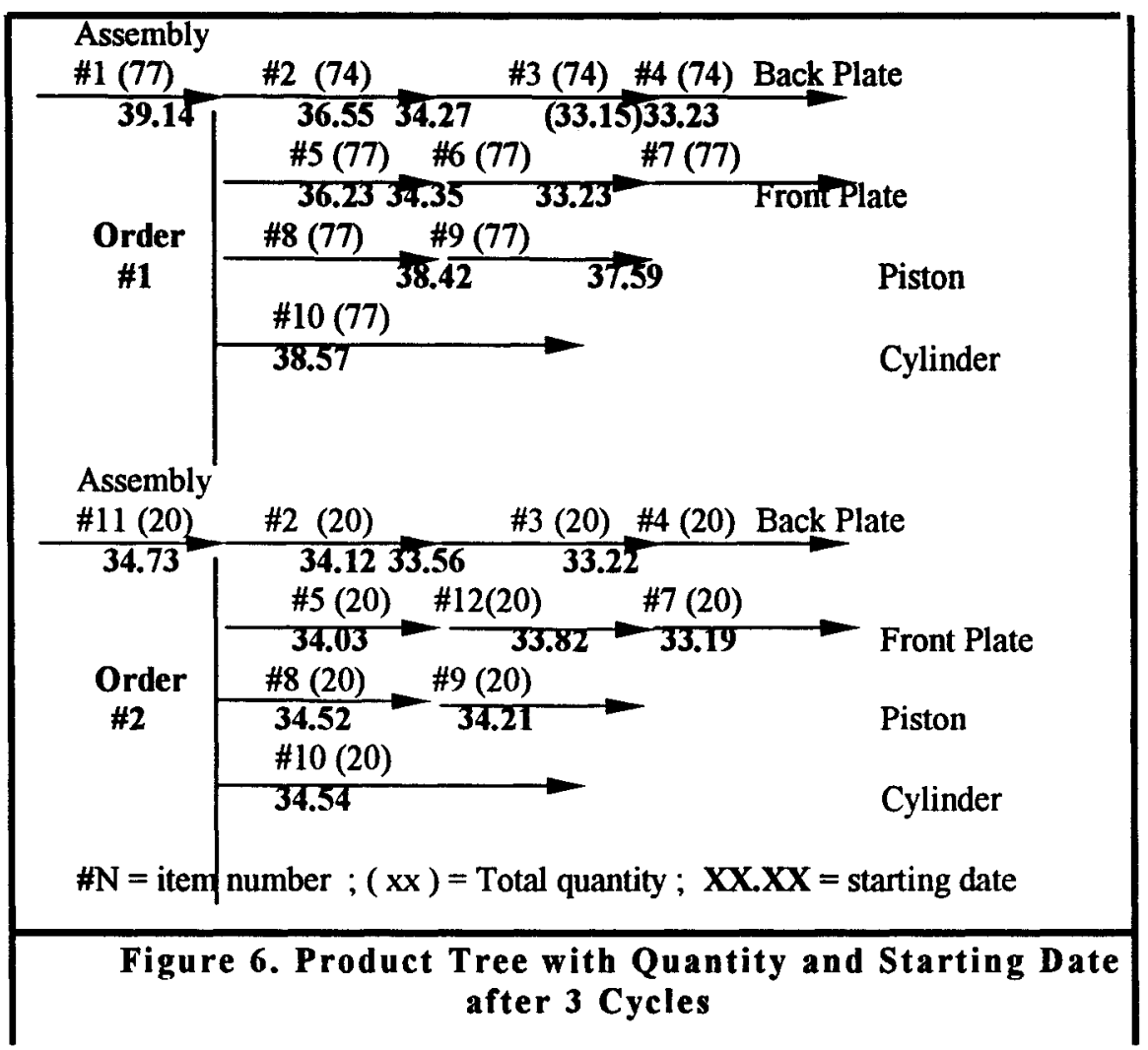

Its last operation is on machine \#4 for one period; it is loaded on period \#5. Then Machine \#3 for one period, and is loaded in period \#6. Next operation calls for machine \#5 for two periods. The available space is on period \#14. The loading process continues going through all the items and orders. The resulting loading scheme is shown in Figure 7a, which deals with the unaltered routing. It takes 23 periods to fill up the 2 orders. The matrix method is a dynamic system, and the routing is regarded as a variable. When the required machine is not idle at the desired period, the matrix will look for an alternative. In the case of item $\#$, the work can be done by machine $\# 10$, which is idle. For item \#6 the matrix system will look for an alternative process. The proposed machine is machine \#6. 


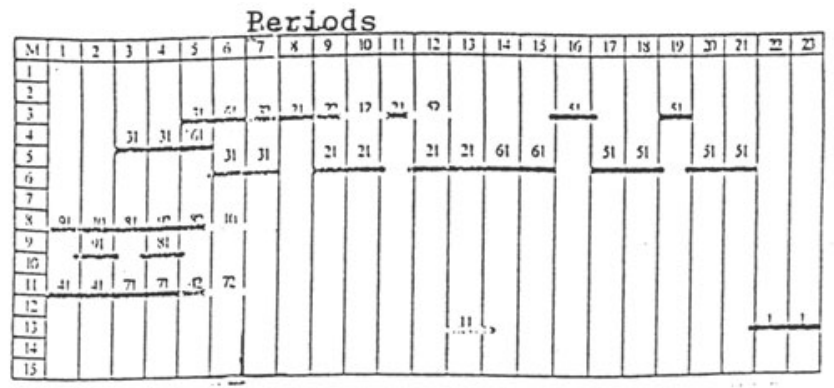

a. Minimum cost with unaltered process

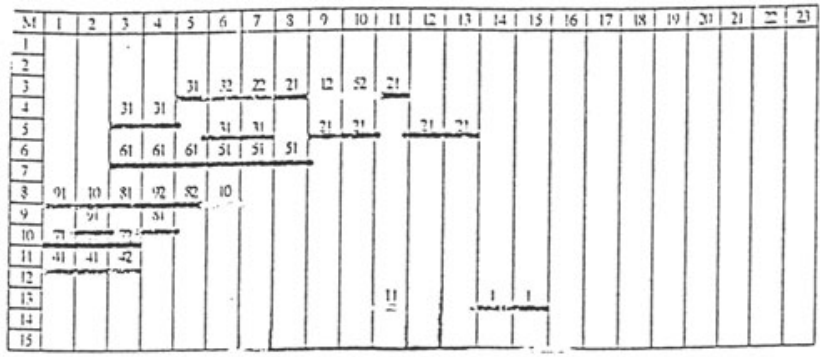

b. Minimum cost with local improvement

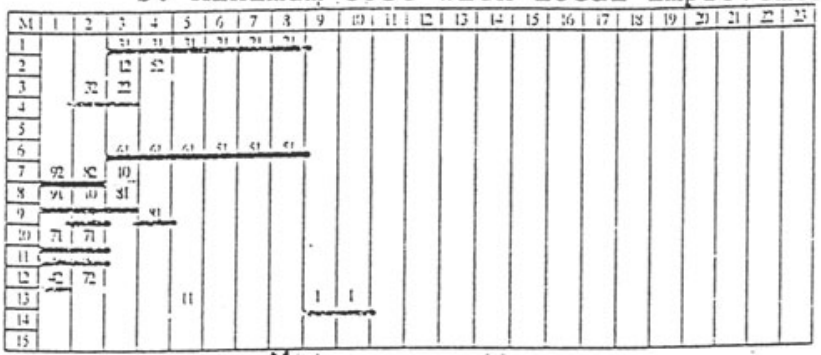

c. Minimum cost with total improvement

Figure 7 : Load Profile of Machine Loading

Machine \#6 is idle. By choosing this alternative, the cost of machining the part will increase from 12.01 to 16.38 . It was decided that this increase in cost is acceptable. Similar logic and procedures are applied to all other items. By introducing the concept of dynamic routing, the two orders can be finished after 15 periods, instead of 23 periods as shown in Figure $7 \mathrm{~b}$. The load profile in Figure $7 \mathrm{~b}$ uses the first alternative. If an additional reduction of the delivery date is required, other alternatives can be employed, like the ones shown in Figure 7c. In this case, the two orders can be finished within 10 periods. The machine loading cycle of those two orders takes 4 seconds on a $33 \mathrm{MHz}$ PC computer.

Using the maximum production optimization criteria instead of the minimum cost optimization criteria, as the retrieved process plan, does not make much difference. It is clear that for each part, the best machine has been selected, i.e. machine \#1. As a result of this, a long queue of work piles up at machine \#1. 


\section{CONCLUSIONS}

The manufacturing process is flexible by nature. However, the manufacturing practice developed over the years, characterized by separated departments and manual planning and management methods, has led to complicated and rigid procedures. Especially in small batch manufacturing, present practice is still merely based on traditional planning and management methods. As an example, capacity plans of a finite number of resources as a procedure preceding scheduling, is hardly applied in industry. However, in the era of the computers and global competition in terms of cost and time to market, the manufacturing methods must be adapted to the new tools and demands. The matrix method presents the basis for an overall planning method, covering the needs of company management strategic planning, factory planning, operations management planning and production. Planning on all these levels can be based on the same basic principle, the matrix. The manufacturing process is composed of many disciplines, were each calling for a particular expertise. The basic concept of the matrix method is that decisions should be made only by the various experts in their own field and at the moment of execution. No one should make decisions that are out of his field of expertise and that may effect other disciplines and disturb the course of the logic decision making. This may seem contradictory with the present trend of integration of manufacturing processes, but it is not. The first step in any true integration process must always be the entanglement of (non-transparent) relations and separation of concerns in order to be able to design transparent processes.

Process planning is not an execution task. Process planners should only prepare data for the production planners. The process planner is an expert in technology, but not an economist, neither business administrator nor a production manager. However, process-planning decisions effect the level of competitiveness of the company. Communication and information transfer between disciplines should merely be of the nature of basic information, alternatives, etc., facilitating the separation of decisions and making subsequent decision making possible without negatively effecting the overall performance (which creates true flexibility). In this context, the matrix method provides an effective tool. It presents many possible process plans without making a decision. Each discipline may use the matrix in the manner it suits best. Company management may use the matrix for resource planning or for rating the company's level of competitiveness. The factory management may use the matrix for negotiation with customers regarding cost and delivery dates. The production management may use the matrix for stock allocation and capacity planning. The shop floor management can use the matrix for scheduling and job release. All the disciplines can work with the same information.

The solution of the matrix is done in seconds on a PC. Therefore, there is no need to store decisions. Decisions can be reproduced much faster by the matrix method then by storage and retrieval systems. The administration becomes simpler and more efficient as the amount of document storage is reduced. The problem of keeping track of versions, and releases is eliminated. Furthermore, decisions may 
be adapted to the dynamic situation of the business. The flexibility and dynamics introduced by the matrix method improves significantly production management. It has been shown that delivery dates may be shortened drastically using the matrix method. The matrix method works with the $b$ method all the time. This feature permits the modification of the production plan for a whole product instead of for an individual item. When disruptions occur in one item, the system adjusts the working plan for all items on the product tree. The results of the experimental prototype of the computer program, written in Q-basic, proves that the matrix method is a realistic one. Running two orders with 10 parts each and in an assumed plant with 15 machines, it takes about 20 seconds to perform stock allocation and capacity planning on a $33 \mathrm{MHz}$ PC.

The additional degree of freedom introduced by the matrix method, raises many operational options. In this work, certain decisions were made before hand, such as, using the minimum cost criteria as the initial working process plan. The reason is that it leaves spare time for rush orders, or in the case of disruptions, moving to maximum production optimization criteria. The critical path was defined by the path in the product tree for which the starting date is the lowest one. However, many other options are possible normally:

the product that uses a bottleneck machine,

the product with the minimum quantity required,

the product for which the penalty for not meeting the delivery date is the greatest,

the part with the maximum number of operations,

the product with the least slack,

the product that does not have an alternate process.

The work so far, proved that the objectives around the matrix method are feasible.

Future plans are, to carry the research further, considering more options and coming up with rules, that can be embedded in the program.

\section{REFERENCES AND BIBLIOGRAPHY:}

Cho, H., Derebail, A., Hale, T., Wysk, R.A. (1994) A formal approach to integrating CAPP and SFC, Transactions of the ASME, Department of Industrial Engineering, Texas A\&M University, Texas, USA, 1994, vol. 116, p.108-116.

Detand, J., (1993), A CAPP system generating Non-Linear Process Plans, Ph.D. Thesis, KU Leuven, October (ISBN 90-73802-23-7)

Halevi G., (1980). The Role of Computers in Manufacturing Processes, JohnWiley \&Sons, NY

Halevi, G. (1993). The magic matrix as a smart scheduler, Computer in Industry $21,1993,245-253$ 
Halevi G., (1995). Principles of process planning - A logistic approach. Chapman \& Hall, London

Halevi, G. (1997). The magic matrix as a smart machine evaluator, International Journal of Production Planning \& Control, Vol. 8, No. 4

Halevi, G. (1997). The magic matrix as a smart resource planner. International Journal of Reduction Engineering and Computers, Vol.1, No. 1, PP- 21-28

Halevi, G. (1996). Computerized benchmarking as a deduction of practical industrial project, Modeling techniques, Business Process ReEngineering(BPR) and Benchmarking, Ed. Guy Doumeingts \& J. Browne, Chapman \& Hall 1997 , pp. 359-374

Huang, S.H., Zhang, H.C., Smith, M.L. (1995). A progressive approach for the integration of Process Planning and Scheduling, IIE Transactions, Department of Industrial Engineering, Texas Tech. University, Lubbock, Texas, USA, vol. 27, p. 456-464.

Kempenaers, J., Pinte, J., Detand, J., Kruth, J.-P., (1996). A collaborative process planning and scheduling system, Advances in Engineering Software, Vol. 25, p. 3-8.

Laliberty, T.J., et al..(1996), A blackboard architecture for integrated process planning/production scheduling, Proceedings of ASME Design for Manufacturing, August

Larsen, N.E., Clausen, J., (1992). Applied methods for integration of process planning and production control, Manufacturing International, pp.349-364.

Nasr, N., Elsayed, E.A.,(1990) Job shop scheduling with alternative machines, Int. J. Prod. Res., Vol. 28, No. 9, p. 1595-1609

Seo ,Y., Egbelu, P.J., (1996), Process plan selection based on product mix and production volume, Int. J. PROD. RES, Vol. 34, No. 9 2639-2655

Tiemersma, J.J., Kals, H.J.J..(1990). A real-time monitoring and control system for small batch part manufacturing. Proc. of the ASME ManufacturingInternational ' 90 conference. Atlanta USA, March 1990, pp. 41-48.

Tiemersma, J.J.,(1992). Shop Floor Control in small Batch Part Manufacturing, Ph.D. Thesis, University of Twente, Enschede, The Netherlands, 1992

Tonshoff, H.K., Beckendorff, U., Andres, N., (1989) FLEXPLAN: A Concept for Intelligent Process Planning and Scheduling. The CIRP International Workshop, Hannover, Germany, September 1989.

\section{BIOGRAPHY}

Gideon Halevi received his M.Sc in Mechanical Engineering from the University of Pennsylvania (1959) and his Doctor of Science in Technology from the Technion (1973). He is as adjunct professor at the Technion (1981), teaching mostly at the graduate school on CAD/CAM and Robotics. For more than 30 years he has been active in industry in production development, Combine Technical 
Operations, heading the corporate computing center and as manager and director of the CAD/CAM Research and Development Center. He has developed the All Embracing Technology, which was published in his book "The Role of Computers in Manufacturing Processes (Wiley 1980). He developed the process planning matrix system, which was published in his book "Principles of Process Planning A logical approach" Chapman \& Hall. 1994 . He is an active member of the CIRP, Israeli representative to IFIP TC5, a member of IFIP WG5.3 and WG5.7, past chairman of SME chapter 319 and past chairman of IPICS.

\section{H.J.J. Kals}

Prof.Dr.ir. H.J.J.Kals is the Director of the Center of Integrated Manufacturing \& development, CIPV. He is also the Head of the Laboratory of Production and Design Engineering at the University of Twente, Netherlands.

$\mathrm{He}$ is the president of the CIRP - International Institution of Production Engineering Research. 